\title{
Prevalence of Post-traumatic Stress Disorder Symptoms Among Psychiatric Patients during the COVID-19 Pandemic
}

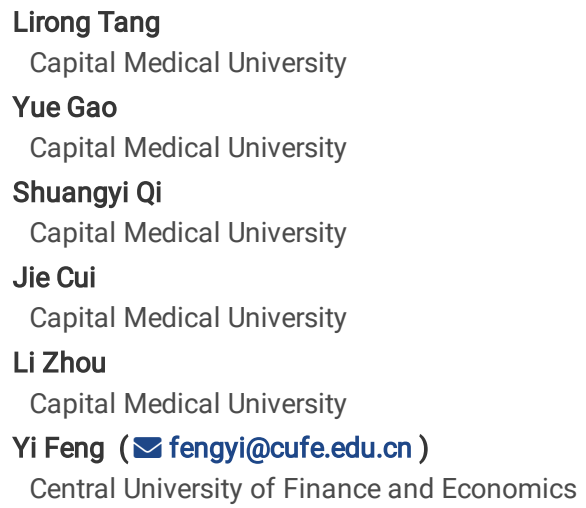




\section{Abstract}

Background: The outbreak of the COVID-19 pandemic has caused extensive public health concerns, posing significant challenges to healthcare services. One particular area of concern is the mental health of psychiatric patients, who are often a neglected group. The aim of this study was to investigate the prevalence of, and associated factors for symptoms of post-traumatic stress disorder (PTSD) among psychiatric patients in China during the COVID-19 pandemic.

Methods: Self-reported questionnaires were distributed to patients in four psychiatric hospitals in Beijing, China, between April $28^{\text {th }}$ and May $30^{\text {th }}, 2020$. Information regarding sociodemographic characteristics, COVID-19 related factors, support, psychosomatic factors, and PTSD symptoms was collected data using a series of scales, such as the Impact of Event Scale-Revised, the 7-item Generalized Anxiety Disorder Scale, the 9-item Patient Health Questionnaire depression scale, and so on. Multivariate regression was used to identify factors related to PTSD symptoms.

Results: A total of 1,055 psychiatric patients were included in the final sample. The prevalence of PTSD symptoms was $41.3 \%$. Hierarchical linear regression demonstrated that fear of the pandemic and anxiety were shared associated factors for both symptoms of PTSD and its subscales. Additionally, age was an associated factor for the total PTSD $(\beta=0.12, p<0.01)$, intrusion $(\beta=0.18, p<0.001)$, and avoidance $(\beta=0.1, p<0.05)$ symptoms; depression was an associated factor for the total PTSD s $(\beta=0.13, p<0.001)$, intrusion $(\beta=0.11, p<0.01)$, and hyperarousal $(\beta=0.19, p<0.001)$ symptoms.

Conclusions: The prevalence of PTSD symptoms was high among psychiatric patients during the COVID-19 pandemic in China. This study found that age, fear of the pandemic, anxiety and depression are significant associated factors of PTSD symptoms in psychiatric patients during the pandemic. We call for higher awareness and introduction of PTSD interventions to relieve the psychological stress in these patients.

\section{Background}

The COVID-19 pandemic has had a substantial impact on many aspects of the physical and mental health of the population worldwide [1]. Psychiatric symptoms have been increasing in both the general population and in patients with the infection during the pandemic [2-4]. Psychiatric patients, who are often a neglected group, have also encountered mental health problems during the pandemic, even if not infected with COVID-19 [5]. Psychiatric patients with severe mental illness, including affective and schizophrenia spectrum disorders, are at a higher risk of negative mental health outcomes related to the pandemic [6]. There are indications of worsening psychiatric symptoms among patients with pre-existing psychiatric disorders [7, 8]. Some experts have speculated that the COVID-19 pandemic might be negatively effecting individuals with pre-existing mental disorders [9-11]. With 16.6\% lifetime prevalence of mental disorders among adults in China [12], millions of psychiatric patients need to be concerned, as they may face barriers when seeking help and timely management of their mental health condition during the pandemic [13]. However, little appears to be known about the pandemic's impact on patients with preexisting psychiatric disorders [14].

Post-traumatic stress disorder (PTSD) is caused by exposure to actual or threatened death, serious injury or sexual violence [15]. There are three main types of symptoms: intrusion symptoms associated with the traumatic events (such as intrusive memories, recurrent distressing dreams, intense or prolonged psychological distress, dissociative reactions, and marked physiological reactions), persistent avoidance symptoms (including avoidance of distressing memories, thoughts or feelings, and numbing of responsiveness), and hyperarousal symptoms (including irritable behavior, angery outbursts, problems with concentration, hypervigilance, and exaggeratedstartle response) [16]. Individuals with PTSD are generally at higher risk of suicide [17]. Long-term exposure to stress may worsen pre-existing chronic health conditions, accelerate the disease's progression, or increase financial burden on patients [18]. Some experts consider PTSD as a secondary effect of the pandemic [17], during which many people are reporting numbness, stiffness, high vigilance, and other psychiatric symptoms [18]. Studies on COVID-19 revealed that PTSD can occur during and after the infectious diseases [19]. The prevalence of PTSD symptoms ranged from 7-53.8\% in the general population during the COVID-19 pandemic in China, Spain, Italy, Iran, the US, Turkey, Nepal, and Denmark [20]. A meta-analysis including 68 independent samples and sub-samples indicate that the PTSD prevalence was $21.94 \%$ during the COVID-19 pandemic, and pandemic-affected groups have significantly higher PTSD prevalence compared to the general population under normal circumstances [21]. A systematic review of the relationship between the COVID-19 pandemic and mental health consequences found that mental health issues in COVID-19 patients present a high level of post-traumatic stress symptoms (96.2\%) [14]. Previous psychiatric disorders displayed suggestive evidence of increasing the risk of PTSD [22]. The onset of PTSD symptoms can make the psychiatric disorder itself more complex and difficult to treat, leading to a greater disease burden [23]. Therefore, clinical doctors need to increase the awareness and importance of PTSD symptoms in psychiatric patients. However, until now there is no research on the prevalence of PTSD symptoms among psychiatric patients during the COVID-19 pandemic.

The causes of PTSD are not fully understood, and whether people who have experienced the same traumatic event develop PTSD is related to sociodemographic characteristics and pre -, peri-, and post-traumatic factors, which interact in complex ways [22]. A systematic review of 54 studies on PTSD found that six pre-traumatic predictors of PTSD included: cognitive level, coping styles; personality characteristics, psychopathology, psychophysiological factors, and socio-ecological factors [24]. Variables related to coping strategies and social/family support showed evidence as PTSD associated factors [22, 25]. All potential consequences of trauma (i.e., symptoms of anxiety, avoidance, or depression) had evidence as post-trauma risk factors [22]. In previous literature, a number of risk and protective factors for PTSD have been identified, however, these findings have not always been consistent [26], inconsistency may reflect unrecognized or unaccounted sources of genuine heterogeneity or biases.

The aim of this study was to examine the prevalence of PTSD symptoms among psychiatric patients during the COVID-19 pandemic and to identify associated factors for PTSD symptoms and its subscales. We assume that due to their susceptibility and vulnerability to crisis, the prevalence of PTSD symptoms among psychiatric patients might be higher than that of the general population during the pandemic [10, 11]. Based on previous study, in addition to sociodemographic characteristics and COVID-19-related factors, we used psychosomatic factors from the perspective of psychological factors (i.e., loneliness, anxiety, and depression), somatic factors (i.e., quality of life, sleep quality), and social ecological factors (i.e., social support) as possible

Page $2 / 12$ 
associated factors for PTSD symptoms [24, 27]. Above all, we hypothesized as follows: (1) The prevalence of PTSD symptoms among psychiatric patients will be higher than that of the general population during the pandemic. (2) Demographic characteristics of the psychiatric patients, such as age and gender, will be significantly associated with the PTSD symptoms. (3) COVID-19 related factors, such as fear of the pandemic, increased pressure by pandemic, will be associated factors with PTSD symptoms. (4) Psychosomatic factors (i.e., loneliness, quality of life, sleep quality, anxiety, and depression) will be significant associated factors with PTSD symptoms among psychiatric patients.

\section{Methods}

\section{Participants and Procedures}

This cross-sectional survey was conducted from April 28 and May 30, 2020. Cluster sampling was used to construct the sample. A questionnaire was distributed by three psychiatrists to all patients in four psychiatric hospitals located in different districts of Beijing, China. There were 1,104 participants in total. The three psychiatrists came from the four different hospitals, and one of them worked in two hospitals. Of these, 550 (49.8\%) participants were from a tertiary specialised hospital, 279 (25.3\%) were from a second-level psychiatric hospital, and 275 (24.9\%) were from two community psychiatric hospitals. The inclusion criteria were as follows: able to write; aged 18-60 years; diagnosed with anxiety disorder, major depressive disorder, bipolar disorder, or schizophrenia. All study participants were informed about the purpose of the study and its procedures before taking part. They were also informed that they could refuse to answer any item and withdraw at any time during the study. Each participant signed an informed consent form before completing the questionnaire. 49 participants did not complete the questionnaire and were excluded, leaving data of 1,055 patients available for analysis, with a completion rate of $95.6 \%$. The study protocol was performed in accordance with the Declaration of Helsinki [28]. The Ethics Committee of the Beijing Anding Hospital affiliated to the Medical Capital University has approved this investigation.

\section{Measures}

\section{Sociodemographic characteristics}

Sociodemographic data were collected, including sex, age, education level, marital status, employment status, annual family income, place of residence, medication status during the pandemic, substance use, and living circumstances during the pandemic. Information was also collected on psychiatric diagnoses, including anxiety disorder, major depressive disorder, bipolar disorder, and schizophrenia.

Medication status during the pandemic was recorded as one of three types: on antipsychotic medications, not on antipsychotic medications, and on nonpsychotropic medications. Substance use was assessed by two questions: "During last month, have you ever experienced symptoms of intoxication such as dizziness, headache and drowsiness due to drinking too much?" on a 5-point scale $(1=$ never, 2 = rarely, $3=$ occasionally, $4=$ often; $5=$ almost every day); and "How many cigarettes did you smoke per day on average?" on a 5-point scale (1 = No smoking history, 2 = Smoking 1-5 cigarettes per day, $3=5$-10 cigarettes; $4=10-20$ cigarettes; 5 = more than 20 cigarettes per day), with higher scores indicating more severe substance use. The final score were obtained by the summing the items.

\section{PTSD symptoms}

PTSD symptoms were measured using the 22-item, self-reported Impact of Event Scale-Revised (IES-R) [16], which assesses the severity of subjective distress caused by traumatic events. Its items are categorised into the three symptom subdomains of intrusion, avoidance, and hyperarousal, and each item is rated on a 5-point scale $(0=$ not at all; $4=$ extremely). The IES-R has been validated, including in China [29], for research regarding health-related trauma, such as that associated with severe acute respiratory syndrome [30, 31]. We considered the cut-off score was 24 in the present study based on previous research [16]. We calculated the composite score for PTSD (Cronbach's $a=0.93$ ) and its symptom subscales (Cronbach's $a=0.84-0.84$ ).

\section{COVID-19 related factors}

COVID-19 related factors includeed fear of the pandemic, increased pressure by pandemic, clinical treatment during pandemic, mental health guidance during pandemic, and medication barriers due to pandemic.

Fear of the pandemic was assessed by two questions: "In the last week, were you worried about getting infected with COVID-19?" and "Do you wash your hands excessively for fear of getting infected with the virus?", both on a 5-point scale ( $1=$ never, 2 = rarely, $3=$ occasionally, $4=$ often; $5=$ almost every day), with higher scores indicating stronger concern. The final values were obtained by the average score. Increased pressure by pandemic was assessed by two questions: "Is your medication status greatly affected by the outbreak? ", and "Did your mental state fluctuate during the outbreak?" both on a 5-point scale with higher scores indicating much less affection and fluctuation. Clinical treatment during pandemic was assessed by one question: "Did you see a psychiatrist during the outbreak?", requiring a "yes" or "no" answer. Mental health guidance during pandemic was assessed by one question: "Did you receive mental health services during the outbreak?", again with a yes or no answer possibility. Medication barriers due to pandemic was also assessed by one question: "Has your access to medicines been affected during the outbreak?" on a 6-point scale, with answers ranging from $1=$ never to $6=$ very often, with higher scores representing more severe medication barriers.

\section{Psychosomatic factors}

Psychosomatic factors include loneliness, quality of life, sleep quality, anxiety, and depression in the present study.

Anxiety was evaluated using the 7-item Generalized Anxiety Disorder Scale (GAD-7) [32], which assesses the frequency of anxiety symptoms in the past two weeks on a 4-point scale $(0=$ not at all; $3=$ nearly every day). The Chinese version of the GAD-7 has been validated $(a=0.90)$, and we considered 5 to be of the clinical cut-off score based on previous research. Cronbach's a was 0.95 in the present study.

Page 3/12 
Depressive symptoms were measured by the 9-item Patient Health Questionnaire (PHQ-9) [33], which determines the frequency of depressive symptoms in the past two weeks on a 4-point scale ( $0=$ none, 1 = on a few days, $2=$ on more than half of the days; $3=$ almost every day). The Chinese version of the PHQ-9 has been validated (Cronbach's a $=0.94$ ) [34], and we considered 5 to be of clinical cut-off score based on previous research [35].

Loneliness was assessed by the question "Do you feel lonely?" on a 5-point scale ( 1 = never, 2 = rarely, $3=$ occasionally, $4=$ often; $5=$ almost every day), with higher scores representing more severe loneliness. Quality of life was assessed by two questions: "How do you feel about your quality of life? " and "Are you satisfied with your present state of health? " on a 5-point scale (1 = extremely unsatisfactory, 2 = rarely not satisfied, $3=$ not satisfied or dissatisfied; $4=$ very satisfied, 5 = extremely satisfactory), with higher scores indicating higher quality of life and greater satisfaction. We obtain the final score by summing the items, with Cronbach's a of 0.76. Sleep quality was assessed by the following three items according to the Pittsburgh Sleep Questionnaire (PSQI-PT) [36]: "How many hours of sleep have you usually been getting per night during the pandemic? ", "Do you take medication to help you sleep during the pandemic? ", and "In general, what do you think of your ability to sleep during the pandemic? " The responses were evaluated using a 5-point scale, with higher scores indicating worse quality of sleep. We obtained the a composite score by adding the items, with Cronbach's a of 0.52 .

\section{Support}

Support was assessed by the level of agreement with the following six statements: (1) "During the pandemic, certain people were always besides me when I encounter problems"; (2) "During the pandemic, I was able to share happiness and sadness with certain people"; (3) "During the pandemic, I was able to obtain help from my family "; (4) "During the pandemic, I was able to obtain emotional help and support from my family when needed"; (5) "I was able to obtain help from my friends during the pandemic"; and (6) "My friends shared happiness and sadness with me during the pandemic". The responses were rated on a 5point scale ( 1 = strongly disagree, 2 = disagree, $3=$ not sure; 4 = agree, 5 = strongly agree) [37]. The composite scores of social support, family support and friends supports were calculated by the sum scores of item (1), (2), item (3), (4) and item (5), (6), respectively (Cronbach's a = 0.75-0.87).

\section{Data analysis}

The sample's characteristics are presented as the mean \pm standard deviation for continuous variables, and as the percentage for categorical variables. Hierarchical linear regression models were used to identify factors related to the PTSD symptom subscales. In Step 1, we entered the sociodemographic variables into the model, before adding COVID-19 related factors in Step 2. In Step 3, we added support and in Step 4, we included psychosomatic factors. An increasing $R^{2}$ value further confirmed the importance of the independent variables regarding the dependent ones. One-way variance analysis and post-hoc tests were used to evaluate PTSD symptoms according to the underlying diagnosis. The statistical analyses were performed using the IBM SPSS Statistics software version 20.0 and R software version 3.6.1. All tests were two-tailed. A p-value less than 0.05 was considered statistically significant.

\section{Results}

\section{Sociodemographic and clinical characteristics}

A total of 1,055 psychiatric patients completed the survey. As shown in Table 1, the average age of the participants was $37.15(S D=13.21)$ years. The characteristics that represented a majority of the patients were as follows: female (65.5\%), a lower education level (62.2\%), unmarried/others (52.6\%), living in an urban area (88.4\%), annual family income lower than 150,000 CNY (71.9\%), and not infected with COVID-19 (93.9\%). All study participants had a preexisting diagnosis (35.4\% with anxiety disorder, $26.7 \%$ with major depressive disorder, $17.6 \%$ with bipolar disorder, and $20.3 \%$ with schizophrenia). During the COVID-19 pandemic, almost half of these patients reported that they did not seek mental health services (47.7\%). More than half of the sample were taking psychiatric medicine (57.3\%) but reported significant COVID-19-related barriers to continuing treatment. 
Table 1

Demographic and clinical characteristics of the study sample $(N=1,055)$

\section{Variables}

Mean Age (SD)

Sex

Male

Female

Education level

Junior school or lower

High school

College or above

Employment status

Full-time

Part-time

Unemployed

Retired

Student

Marital status

Married

Unmarried

Others/Not clear

Family annual income

$<30,000$

$30,000 \sim 60,000$

$60,000 \sim 150,000$

$150,000 \sim 250,000$

$>250,000$

Residence place

Urban

Rural

Residence status during the pandemic

Live alone

Live together with others

Live in hospital

Others

Infection with COVID-19

No

Yes

Mean Substance use (SD)

Medication status during pandemic

On psychotropic medications (yes)

Not on any medications (no)
Number

37.15

364

34.5

691

65.5

183

17.3

474

44.9

398

37.7

419

61

283

39.7

142

150

5.8

26.8

13.5

14.2

47.4

500

43.1

455

100

9.5

253

24.0

210

19.9

295

28.0

152

14.4

145

13.7

933

88.4

122

11.6

110

10.4

878

83.2

62

5.9

5

0.5

991

93.9

64

6.1

1.36

(0.66)

604

57.3

214

20.3

Note. The unit of annual income is CNY yuan. Categorical variables were presented in the form of mean and standardized deviation, and continuous variables were presented in the form of number and proportion. 


\begin{tabular}{|c|c|c|}
\hline Variables & Number & Percent (\%) \\
\hline On non-psychotropic medications (no) & 237 & 22.5 \\
\hline \multicolumn{3}{|l|}{ Psychiatric diagnosis } \\
\hline Anxiety disorder & 373 & 35.4 \\
\hline Major depressive disorder & 282 & 26.7 \\
\hline Bipolar disorder & 186 & 17.6 \\
\hline Schizophrenia & 214 & 20.3 \\
\hline Mean Fear of pandemic (SD) & 2.46 & $(0.67)$ \\
\hline Mean Increased pressure by pandemic (SD) & 2.23 & $(0.93)$ \\
\hline \multicolumn{3}{|l|}{ Psychiatric treatment during pandemic } \\
\hline Yes & 652 & 61.8 \\
\hline No & 403 & 38.2 \\
\hline \multicolumn{3}{|l|}{ Mental health guidance during pandemic } \\
\hline Yes & 552 & 52.3 \\
\hline No & 503 & 47.7 \\
\hline Mean Medication barrier during pandemic (SD) & 2.15 & $(1.02)$ \\
\hline \multicolumn{3}{|l|}{ Supports } \\
\hline Mean Social support (SD) & 4.43 & $(1.34)$ \\
\hline Mean Family support (SD) & 4.98 & $(1.48)$ \\
\hline Mean Friends support (SD) & 4.71 & $(1.41)$ \\
\hline \multicolumn{3}{|l|}{ Psychosomatic factors } \\
\hline Mean Loneliness (SD) & 2.39 & $(1.31)$ \\
\hline Mean Quality of life (SD) & 3.04 & $(0.89)$ \\
\hline Mean Sleep quality (SD) & 2.52 & $(0.59)$ \\
\hline Generalized anxiety symptoms ( $\geq 5$ ) & 540 & 51.0 \\
\hline Depressive symptoms ( $\geq 5$ ) & 604 & 57.3 \\
\hline PTSD symptoms ( $\geq 24)$ & 436 & 41.3 \\
\hline
\end{tabular}

\section{Prevalence of PTSD symptoms}

Four hundred and thirty-six patients (41.3\%) had PTSD symptoms, which were then investigated according to underlying psychiatric diagnosis (anxiety disorder, major depressive disorder, bipolar disorder, or schizophrenia). There was no significant between-group difference in the total score for PTSD ( $F$ $(3,1049)=2.22, p=0.084)$, the intrusion symptom $(F(3,1051)=1.16, p=0.324)$, or the avoidance symptom $(F(3,1051)=0.394, p=0.758)$. Patients with major depressive disorder had the highest mean score $(14.08 \pm 5.30)$ for the hyperarousal symptom, significantly higher than the anxiety group $(p<0.05)$ and the schizophrenia group $(p<0.05)$ (see Figure 1$)$.

\section{Factors associated with PTSD symptoms}

As shown in Table 2, the hierarchical linear regression demonstrated that fear of the pandemic and anxiety were shared associated factors for both PTSD symptoms and its subscales. Additionally, age was an associated factor for total PTSD score $(\beta=0.12, p<0.01)$, intrusion $(\beta=0.18, p<0.001)$, and avoidance $(\beta=0.1, p<0.05)$; depressive symptoms were associated factors for total PTSD score $(\beta=0.13, p<0.001)$, intrusion $(\beta=0.11, p<0.01)$, and hyperarousal $(\beta=$

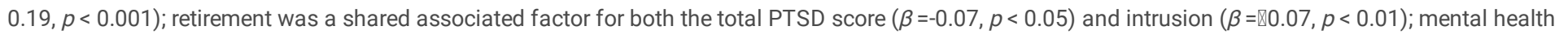
guidance during the pandemic was a unique associated factor for PTSD symptoms $(\beta=\llbracket 0.05, p<0.05)$; medication status during the pandemic $(\beta=\llbracket 0.08, p<$ $0.05)$ and psychiatric treatment $(\beta=0.06, p<0.05)$ were unique associated factors for intrusion; quality of life was an unique associated factor for avoidance $(\beta=0.09, p<0.05)$; urban residence $(\beta=0.04, p<0.05)$, increased pressure $(\beta=0.09, p<0.05)$, family support $(\beta=0.05, p<0.05)$, friends support $(\beta=\llbracket 0.05, p<$ $0.05)$, loneliness $(\beta=0.06, p<0.05)$, and sleep quality $(\beta=0.06, p<0.01)$ were all unique associated factors for hyperarousal. 
Table 2

Hierarchical linear regression coefficients for different PTSD symptoms $(N=1,055)$

\begin{tabular}{|c|c|c|c|c|c|c|c|c|c|c|c|c|c|}
\hline \multirow[t]{2}{*}{ Variables } & \multicolumn{4}{|l|}{ PTSD } & \multicolumn{4}{|c|}{ Intrusion subscale } & \multicolumn{4}{|c|}{ Avoidance subscale } & \multirow{2}{*}{$\begin{array}{l}\text { Hyperarou } \\
\text { Model } \\
1\end{array}$} \\
\hline & $\begin{array}{l}\text { Model } \\
1\end{array}$ & $\begin{array}{l}\text { Model } \\
2\end{array}$ & $\begin{array}{l}\text { Model } \\
3\end{array}$ & $\begin{array}{l}\text { Model } \\
4\end{array}$ & $\begin{array}{l}\text { Model } \\
1\end{array}$ & $\begin{array}{l}\text { Model } \\
2\end{array}$ & $\begin{array}{l}\text { Model } \\
3\end{array}$ & $\begin{array}{l}\text { Model } \\
4\end{array}$ & $\begin{array}{l}\text { Model } \\
1\end{array}$ & $\begin{array}{l}\text { Model } \\
2\end{array}$ & $\begin{array}{l}\text { Model } \\
3\end{array}$ & $\begin{array}{l}\text { Model } \\
4\end{array}$ & \\
\hline \multicolumn{14}{|c|}{ Socio-demographics characteristics } \\
\hline Sex (male) & -0.05 & -0.00 & -0.01 & 0.01 & -0.03 & 0.02 & 0.02 & 0.03 & -0.04 & -0.01 & -0.01 & 0.00 & $-0.07 *$ \\
\hline Age & -0.00 & 0.03 & 0.05 & $0.12^{\star \star}$ & 0.08 & $0.10 *$ & $0.16^{*}$ & $0.18^{\star \star \star}$ & 0.02 & 0.04 & 0.05 & $0.10^{*}$ & $-0.11^{*}$ \\
\hline Education & -0.04 & -0.02 & -0.00 & 0.01 & -0.02 & 0.00 & 0.01 & 0.03 & -0.04 & -0.02 & -0.01 & 0.00 & -0.04 \\
\hline $\begin{array}{l}\text { Employment } \\
\text { (part-time) }\end{array}$ & $-0.08^{*}$ & $-0.06^{\star}$ & $-0.06 *$ & -0.03 & -0.06 & -0.05 & -0.05 & -0.03 & -0.06 & -0.05 & -0.06 & -0.03 & $-0.08^{*}$ \\
\hline $\begin{array}{l}\text { Employment } \\
\text { (unemployed) }\end{array}$ & -0.04 & -0.01 & -0.03 & -0.00 & -0.06 & -0.03 & -0.04 & -0.03 & -0.02 & 0.01 & -0.00 & 0.02 & -0.04 \\
\hline $\begin{array}{l}\text { Employment } \\
\text { (retired) }\end{array}$ & -0.06 & -0.07 & -0.08 & $-0.07 *$ & -0.06 & -0.07 & -0.07 & $-0.07 *$ & -0.06 & -0.07 & -0.07 & -0.07 & -0.03 \\
\hline $\begin{array}{l}\text { Employment } \\
\text { (student) }\end{array}$ & -0.08 & -0.05 & -0.05 & -0.04 & -0.07 & -0.04 & -0.04 & -0.03 & -0.06 & -0.04 & -0.04 & -0.03 & $-0.09 *$ \\
\hline $\begin{array}{l}\text { Marital status } \\
\text { (unmarried) }\end{array}$ & -0.03 & -0.00 & -0.01 & 0.01 & -0.04 & -0.02 & -0.03 & -0.00 & -0.00 & 0.02 & 0.01 & 0.03 & -0.03 \\
\hline $\begin{array}{l}\text { Marital status } \\
\text { (others) }\end{array}$ & 0.01 & 0.03 & 0.03 & 0.00 & -0.04 & -0.01 & -0.01 & -0.03 & 0.05 & $0.07 *$ & $0.07 *$ & 0.05 & -0.00 \\
\hline Income & -0.02 & 0.01 & 0.01 & -0.04 & -0.01 & 0.01 & 0.02 & -0.02 & -0.04 & -0.03 & -0.03 & -0.05 & 0.01 \\
\hline $\begin{array}{l}\text { Residence } \\
\text { (urban) }\end{array}$ & 0.03 & 0.03 & 0.03 & 0.03 & -0.01 & -0.01 & -0.01 & -0.01 & 0.05 & 0.05 & 0.05 & 0.05 & 0.04 \\
\hline Live (alone) & 0.00 & 0.01 & 0.00 & 0.02 & 0.05 & 0.02 & 0.01 & 0.02 & 0.01 & 0.02 & 0.01 & 0.02 & -0.02 \\
\hline $\begin{array}{l}\text { Live } \\
\text { (hospital) }\end{array}$ & 0.01 & -0.00 & -0.01 & -0.00 & 0.04 & 0.02 & 0.02 & 0.02 & -0.00 & -0.02 & -0.02 & -0.02 & -0.01 \\
\hline Live (others) & $-0.06 *$ & -0.06 & -0.05 & -0.03 & -0.05 & -0.04 & -0.03 & -0.02 & $-0.07 \star$ & -0.06 & -0.05 & -0.05 & -0.05 \\
\hline $\begin{array}{l}\text { Infection } \\
\text { (yes) }\end{array}$ & 0.02 & -0.01 & -0.01 & 0.01 & 0.02 & 0.01 & 0.00 & 0.01 & 0.01 & 0.00 & 0.00 & 0.01 & 0.00 \\
\hline $\begin{array}{l}\text { Substance } \\
\text { use }\end{array}$ & $0.14^{\star \star \star}$ & $0.06 *$ & 0.05 & -0.01 & $0.11^{\star \star}$ & 0.05 & 0.04 & -0.01 & $0.12^{\star \star \star}$ & 0.06 & 0.05 & 0.01 & $0.15^{\star \star \star}$ \\
\hline $\begin{array}{l}\text { Medication } \\
\text { (yes) }\end{array}$ & -0.00 & -0.05 & -0.03 & -0.05 & -0.03 & $-0.08^{*}$ & -0.06 & $-0.08^{*}$ & 0.01 & -0.03 & -0.02 & -0.03 & 0.02 \\
\hline $\begin{array}{l}\text { Diagnosis } \\
\text { (anxiety) }\end{array}$ & 0.04 & 0.06 & 0.05 & 0.05 & 0.02 & 0.03 & 0.03 & 0.02 & 0.06 & 0.07 & 0.06 & 0.07 & 0.04 \\
\hline $\begin{array}{l}\text { Diagnosis } \\
\text { (depression) }\end{array}$ & $0.11^{\star \star}$ & 0.07 & 0.04 & -0.04 & 0.10 & 0.06 & 0.04 & -0.03 & 0.04 & 0.01 & -0.01 & -0.05 & $0.18^{\star \star \star}$ \\
\hline $\begin{array}{l}\text { Diagnosis } \\
\text { (bipolar) }\end{array}$ & 0.07 & 0.01 & -0.00 & 0.01 & $0.06^{*}$ & 0.01 & 0.00 & 0.01 & 0.05 & 0.01 & 0.00 & 0.01 & 0.07 \\
\hline \multicolumn{14}{|c|}{ COVID-19 related factors } \\
\hline Fear of pandem & & $0.27^{\star \star \star}$ & 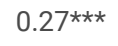 & $0.20 * \star \star$ & & $0.31^{\star \star \star}$ & $0.31^{\star \star \star}$ & $0.25^{\star \star \star}$ & & $0.22^{\star \star \star}$ & $0.22^{\star \star \star}$ & $0.17^{\star \star \star}$ & \\
\hline Increased press & & $0.37^{\star \star \star}$ & $0.32^{\star \star *}$ & 0.04 & & $0.27^{\star \star \star}$ & $0.24^{\star \star \star}$ & 0.01 & & $0.23^{\star \star \star}$ & 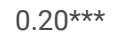 & 0.02 & \\
\hline Psychiatric trea & tment & 0.06 & 0.06 & 0.05 & & $0.07 *$ & $0.07 *$ & $0.06^{*}$ & & 0.05 & 0.05 & 0.05 & \\
\hline Mental health g & uidance & -0.01 & -0.03 & $-0.05^{\star}$ & & 0.01 & -0.00 & -0.03 & & -0.06 & $-0.07 *$ & $-0.08 * \star$ & \\
\hline Medication bar & & $-0.14^{\star *}$ & $-0.12^{\star \star}$ & 0.03 & & $-0.10^{\star}$ & -0.09 & 0.03 & & -0.06 & -0.05 & 0.04 & \\
\hline \multicolumn{14}{|l|}{ Support } \\
\hline Social support & & & -0.04 & -0.04 & & & -0.02 & -0.02 & & & -0.05 & -0.05 & \\
\hline Family support & & & $-0.10 \star \star$ & 0.01 & & & -0.11 ** & -0.02 & & & -0.07 & -0.00 & \\
\hline Friends suppor & & & $-0.09 *$ & -0.03 & & & -0.05 & -0.00 & & & -0.05 & -0.02 & \\
\hline
\end{tabular}

Note. All the regression coefficients in the above table were standardized regression coefficients. ${ }^{\star} p<.05 ;{ }^{* \star} p<.01 ;{ }^{* \star} p<.001$. 


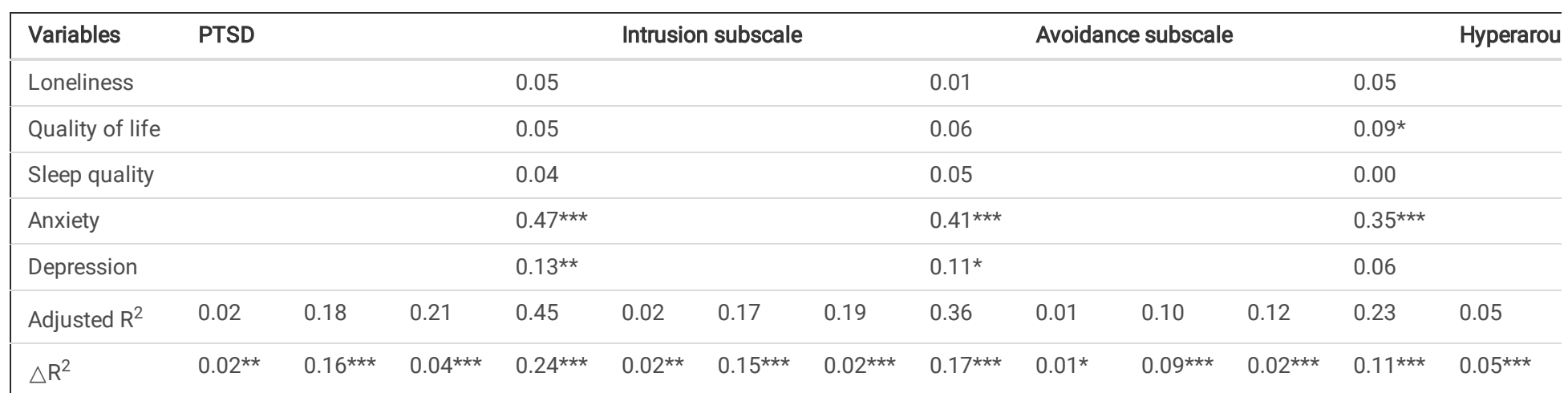

Note. All the regression coefficients in the above table were standardized regression coefficients. ${ }^{*} p<.05 ;{ }^{* \star} p<.01 ;{ }^{* \star \star} p<.001$.

\section{Discussion}

The prevalence of PTSD symptoms (41.3\%) in this study is much higher than the previously reported lifetime prevalence level in the general population (29\%) [38]. A systematic review showed the pooled prevalence of PTSD among participants during the COVID-19 pandemic to be 21.94\% [21]. A study conducted between April 4 and 6, 2020, among the medics working in Wuhan upon their return after work indicated overall prevalence of clinically concerned PTSD symptoms of $31.6 \%$ [39], by the same scale (IES-R). A survey-based cross-sectional study performed from January 29 to February 7, 2020 , in Wuhan, China, showed an estimated PTSD prevalence of $9.8 \%$ [40]. In our study, this prevalence of PTSD was even higher. Exact comparisons are difficult to make because some of the other research have used different measures. However, it is suggested that although Beijing is not an area with the highest risk of COVID-19, the prevalence of PTSD symptoms in psychiatric patients in Beijing is high, indicating the susceptibility of this group. Clinicians must be aware that these patients may experience higher rates and severity of post-traumatic stress disorder than the general population [20].

In this study, there was no significant difference between the severity of the total PTSD score among different patients with mental disorders. This may indicate that patients with different diseases share similar psychological characteristics, including vulnerability and susceptibility, causing similar effects during the COVID-19 pandemic. However, the scores of the PTSD hyperarousal symptoms were higher in patients with major depressive disorder than in those with anxiety disorder or schizophrenia. Evidence suggests that the associations between PTSD and depression are complex, involving bidirectional causality, common risk factors, and common vulnerabilities [23,41,42]. Hyperarousal includes irritability, anger, difficult concentrating, hypervigilance, and a heightened startle response [16]. The results of this study suggested that more attention should be paid to the characteristics of high arousal in patients with major depressive disorder.

The study found evidence for the second hypothesis that demographic characteristics were associated with PTSD symptoms. This study showed that age was an associated factor for the total PTSD score, intrusion, and avoidance. Since the COVID-19 virus is more serious and with higher mortality rate in older people [43], they may have more severe PTSD. Retirement was a shared associated factor for both the total PTSD score and intrusion in the study, indicating that retirement may be a protective factor for PTSD. A possible explanation is that retirees may need to travel less during the epidemic and have higher financial security, therefore being less stressed by the epidemic [1].

The third hypothesis, COVID-19 related factors are associated with PTSD symptoms, was well supported by the data. Fear of the pandemic were shared associated factors for both PTSD symptoms and its subscales. There have been reports of anxiety and fear often co-existing and comorbid with PTSD [44, 45]. Mental health guidance during the pandemic was a unique associated factor, while clinical treatment during pandemic, or medication barriers due to pandemic were not significant associated factors for PTSD symptoms, which might indicate that mental health interventions and resources could help psychiatry patients reduce the stress caused by the epidemic and the incidence of PTSD.

The fourth hypothesis, psychosomatic factors are significant associated factors with PTSD symptoms, was supported by the data. Anxiety symptoms was shared associated factors for both PTSD symptoms and its subscales. Recent neuroscience research suggested that higher sensitivity to anxiety tends to increase the severity of PTSD [46]. Individuals with higher stress/fear levels may become impatient, feel upset or agitated, and experience difficulty relaxing, all of which have a negative impact on PTSD symptoms [47]. Depression symptoms were associated factors for the total PTSD score, intrusion and hyperarousal. As depression is the disorder most commonly associated with PTSD [23,42], people with depressive symptoms may be more likely to develop PTSD, which should be particularly noticed. Quality of life was a unique associated factor for avoidance, implying that patients were more concerned about it. During the epidemic, people's quality of life deteriorated [48]. Self-rated poor health during an outbreak was significantly associated with greater psychological impact and higher levels of stress, according to a study in China [31].

Another prominent finding was that several unique factors were associated with sub

-dimensions of PTSD. Most obviously, urban residence, increased pressure, loneliness, support from friends and sleep quality were all unique associated factor for hyperarousal but not associated with intrusion or avoidance. These results might indicate that there were differences among the related factors of the three dimensions of PTSD, and hyperarousal require unique attention [16]. During an epidemic, isolation policies and inadequate social support can lead to feelings of loneliness [1]. Previous studies showed that isolation can negatively affect mental health [7, 8]. Our findings correlate to those of other studies on the general population. Social support plays a key role in mitigating the risk of mental health problems [49]. The results also demonstrated that support from friends was associated with a lower incidence of hyperarousal symptoms, while support from family might increase patients' hyperarousal symptoms. This 
finding is a reminder that too much unnecessary care from family could increase patients' hyperarousal symptoms. Thus, "moderate" care from friends is necessary for psychiatric patients. These results have great implications for clinicians in predicting and treating patients with high hyperarousal symptoms.

\section{Implications}

To the best of our knowledge, this is the first study to screen for PTSD symptoms in patients with a pre-existing psychiatric diagnosis during the COVID-19 pandemic in Beijing, China. Primarily, the prevalence of symptoms of PTSD among psychiatric patients was not encouraging, arousing attentions from medical staff, related psychologists and mental health centers. Next, this study explored some risk factors (e.g., old age, depressive disorder, fear) and protective factors (e.g., retirement, mental health guidance) for PTSD, providing specific reference and guidance for the psychological prevention and intervention among psychiatric patients in face of the COVID-19 pandemic. Furthermore, this study examined PTSD as well as the three subscales, discriminating the difference in the relationship between PTSD subscales and related psychosomatic factors. The uniqueness of hyperarousal factor provided a theoretical reference for better understanding the structure of PTSD symptoms.

\section{Limitations}

This study has several limitations that should be considered when interpreting its findings. First, it adopted a cross-sectional design, so the way PTSD symptoms in psychiatric patients may change over time is unclear. A longitudinal study is required to identify protective factors and the long-term impacts of PTSD in psychiatric patients during the pandemic. Second, the sample was limited to patients in just four psychiatric hospitals in Beijing, China. Therefore, a nationwide or worldwide multicentre study is needed to provide broader data about PTSD symptoms in psychiatry patients during the COVID-19 pandemic. Finally, no objective biological indicators were included for psychosomatic factors. In further research, other indicators such as peripheral blood, heredity, inflammation, immune and metabolic function markers, or brain imaging are necessary.

\section{Conclusions}

This study found that the prevalence of PTSD symptoms was high among psychiatric patients during the COVID-19 pandemic in China, as well as the associated factors for PTSD symptoms, including socio-demographics and psychosomatic factors, shedding practical implication on the PTSD status among psychiatric patients. We recommended that clinical psychiatrists increase the awareness of PTSD symptoms among psychiatric patients and provide effective mental health interventions for patients to manage those symptoms.

\section{Declarations}

\section{Ethics approval and consent to participate}

Before the survey, all participants were informed of the purpose and procedure of the survey and signed an online informed consent. The study protocol was performed in accordance with the Declaration of Helsinki. The Beijing Anding Hospital affiliated to Medical Capital University Ethics Committee has approved this investigation (No: 2020-10).

\section{Consent for publication}

Not applicable. All participants participated in the survey anonymously.

\section{Availability of data and materials}

The datasets used during the current study are available from the corresponding author on reasonable request.

\section{Competing interests}

The authors declare that they have no competing interests.

\section{Funding}

This study was funded by Beijing Hospitals Authority Youth Programme (Grant No. QML 20191902), Beijing Hospitals Authority Yangfan Programme (Grant No. ZYLX201815), National Major Science and Technology Projects of China (Grant No. 2018ZX10715-005) and Beijing Municipal Administration of Hospitals Clinical Medicine Development of Special Funding Support (Grant No. ZYLX201807).

\section{Author's contributions}

All authors contributed to this study significantly. LT, YF and YG designed the survey; LT, JC and YG collected data; YF analysed and interpreted data; LT and YF drafted the manuscript; YF controlled the quality; All author contributed to revise it. All authors read and approved the final manuscript. 


\section{Acknowledgments}

We acknowledge that Tuozhen Liu helped with data visualization. We also thank all the participants in this study.

\section{References}

1. Pieh C, Budimir S, Probst T. The effect of age, gender, income, work, and physical activity on mental health during coronavirus disease (COVID-19) lockdown in Austria. J Psychosom Res. 2020;136:110186.

2. Poyraz BC, Poyraz CA, Olgun Y, Gurel O, Alkan S, Ozdemir YE, et al. Psychiatric morbidity and protracted symptoms after COVID-19. Psychiatry Res. 2021;295:113604.

3. Liu D, Baumeister RF, Veilleux JC, Chen C, Liu W, Yue Y, et al. Risk factors associated with mental illness in hospital discharged patients infected with COVID-19 in Wuhan, China. Psychiatry Res. 2020;292:113297.

4. Chang MC, Park D. Incidence of Post-Traumatic Stress Disorder After Coronavirus Disease. Healthcare (Basel). 2020;8(4).

5. McGowan ML, Norris AH, Bessett D. Care Churn - Why Keeping Clinic Doors Open Isn't Enough to Ensure Access to Abortion. N Engl J Med. 2020;383(6):508-10.

6. Pinkham AE, Ackerman RA, Depp CA, Harvey PD, Moore RC. A Longitudinal Investigation of the Effects of the COVID-19 Pandemic on the Mental Health of Individuals with Pre-existing Severe Mental Illnesses. Psychiatry Res. 2020;294:113493.

7. Fernández-Aranda F, Casas M, Claes L, Bryan DC, Favaro A, Granero R, et al. COVID-19 and implications for eating disorders. European eating disorders review: the journal of the Eating Disorders Association. 2020;28(3):239-45

8. Zhou J, Liu L, Xue P, Yang X, Tang X. Mental Health Response to the COVID-19 Outbreak in China. The American journal of psychiatry. 2020;177(7):574-5.

9. Sergeant. A, Reekum. EAv, Sanger. N, Dufort. A, Rosic. T, Sanger. S, et al. Impact of COVID-19 and other pandemics and epidemics on people with preexisting mental disorders: a systematic review protocol and suggestions for clinical care. BMJ Open. 2020;10:e040229.

10. Yao. H, Chen. J-H, Xu. Y-F. Patients with mental health disorders in the COVID-19 epidemic. Lancet Psychiatry. 2020;7(4):e21.

11. Druss BG. Addressing the COVID-19 Pandemic in Populations With Serious Mental Illness. JAMA Psychiatry. 2020;77(9):891-2.

12. Huang Y, Wang Y, Wang H, Liu Z, Yu X, Yan J, et al. Prevalence of mental disorders in China: a cross-sectional epidemiological study. The Lancet Psychiatry. 2019;6(3):211-24.

13. Xiang YT, Zhao N, Zhao YJ, Liu Z, Zhang Q, Feng Y, et al. An overview of the expert consensus on the mental health treatment and services for major psychiatric disorders during COVID-19 outbreak: China's experiences. Int J Biol Sci. 2020;16(13):2265-70.

14. Vindegaard N, Benros ME. COVID-19 pandemic and mental health consequences: Systematic review of the current evidence. Brain Behav Immun. 2020;89:531-42.

15. Casagrande M, Favieri F, Tambelli R, Forte G. The enemy who sealed the world: effects quarantine due to the COVID-19 on sleep quality, anxiety, and psychological distress in the Italian population. Sleep Med. 2020;75:12-20.

16. McCabe. D, DNP., APRN-BC., GNP. The Impact of Event Scale - Revised (IES-R). 2020.

17. Dutheil. F, Mondillon. L,. VN. PTSD as the second tsunami of the SARS-Cov-2 pandemic. Psychological Medicine. 2020:1-2.

18. Cooke JE, Eirich R, Racine N, Madigan S. Prevalence of posttraumatic and general psychological stress during COVID-19: A rapid review and metaanalysis. Psychiatry Res. 2020;292:113347.

19. Shuja KH, Aqeel M, Jaffar A, Ahmed A. COVID-19 Pandemic and Impending Global Mental Health Implications. Psychiatr Danub. 2020;32(1):32-5.

20. Xiong J, Lipsitz O, Nasri F, Lui LMW, Gill H, Phan L, et al. Impact of COVID-19 pandemic on mental health in the general population: A systematic review. J Affect Disord. 2020;277:55-64.

21. Cenat JM, Blais-Rochette C, Kokou-Kpolou CK, Noorishad PG, Mukunzi JN, Mclntee SE, et al. Prevalence of symptoms of depression, anxiety, insomnia, posttraumatic stress disorder, and psychological distress among populations affected by the COVID-19 pandemic: A systematic review and metaanalysis. Psychiatry Res. 2021;295:113599.

22. Tortella-Feliu M, Fullana MA, Perez-Vigil A, Torres X, Chamorro J, Littarelli SA, et al. Risk factors for posttraumatic stress disorder: An umbrella review of systematic reviews and meta-analyses. Neurosci Biobehav Rev. 2019;107:154-65.

23. Breslau N, Chase GA, Anthony JC. The uniqueness of the DSM definition of post-traumatic stress disorder: implications for research. Psychol Med. 2002;32(4):573-6.

24. DiGangi JA, Gomez D, Mendoza L, Jason LA, Keys CB, Koenen KC. Pretrauma risk factors for posttraumatic stress disorder: a systematic review of the literature. Clin Psychol Rev. 2013;33(6):728-44.

25. Steel. Z, Chey. T, Silove. D, Marnane. C, Bryant. RA, Ommeren. Mv. Association of Torture and Other Potentially Traumatic Events With Mental Health Outcomes Among Populations Exposed to Mass Conflict and Displacement A Systematic Review and Meta-analysis. JAMA 2009;304(5):537-49.

26. Shalev. AY, Gevonden. M, Ratanatharathorn. A, Laska. E, Mei. WFvd, Qi. W, et al. Estimating the risk of PTSD in recent trauma survivors: results of the International Consortium to Predict PTSD (ICPP). World Psychiatry. 2019;18:77-87.

27. Zhang Z, Feng Y, Song R, Yang D, Duan X. Prevalence of psychiatric diagnosis and related psychopathological symptoms among patients with COVID-19 during the second wave of the pandemic. Global Health. 2021;17(1):44.

28. Association WM. World Medical Association Declaration of Helsinki: ethical principles for medical research involving human subjects. Jama. 2013;310(20):2191-4. 
29. Xiao-jun. W, Shi-liang. W, Yan-hong. H, Chen-jie. G, Guo-hua. S, Zhong-xia. S. Reliability and Validity of the Impact of Event Scale-Revised (IES-R) in the Isolated Population under the Epidemic Situation of COVID-19. Chinese Journal of Clinical Psychology. 2020;28(4):760-2.

30. Wu. P, Fang. Y, Guan. Z, Fan. B, Kong. J, Yao. Z, et al. The psychological impact of the SARS epidemic on hospital employees in China: exposure, risk perception, and altruistic acceptance of risk. Can J Psychiatry. 2009;54(5):302-11.

31. Wang C, Pan R, Wan X, Tan Y, Xu L, Ho CS, et al. Immediate Psychological Responses and Associated Factors during the Initial Stage of the 2019 Coronavirus Disease (COVID-19) Epidemic among the General Population in China. Int J Environ Res Public Health. 2020;17(5).

32. Spitzer. RL, Kroenke. K, Williams. JBW, Löwe. B. A Brief Measure for Assessing Generalized Anxiety Disorder. Arch Intern Med. 2006;166(10):1092-7.

33. Kroenke. K, Spitzer. RL. The PHQ-9_ A New Depression Diagnostic and Severity Measure. Psychiatric Annals. 2002;32(9):509-15.

34. Ye. X, Shu. H-L, Feng. X, Xia. D-M, Wang. Z-Q, Mi. W-Y, et al. Reliability and validity of the Chinese version of the Patient Health Questionnaire-9 (C-PHQ-9) in patients with psoriasis: a cross-sectional study. BMJ Open. 2020;10:E033211.

35. Wang W, Bian Q, Zhao Y, Li X, Wang W, Du J, et al. Reliability and validity of the Chinese version of the Patient Health Questionnaire (PHQ-9) in the general population. Gen Hosp Psychiatry. 2014;36(5):539-44.

36. Del Rio Joao KA, Becker NB, de Neves Jesus S, Isabel Santos Martins R. Validation of the Portuguese version of the Pittsburgh Sleep Quality Index (PSQIPT). Psychiatry Res. 2017;247:225-9.

37. Wang Y, Duan Z, Ma Z, Mao Y, Li X, Wilson A, et al. Epidemiology of mental health problems among patients with cancer during COVID-19 pandemic. TransI Psychiatry. 2020;10(1):263.

38. Atwoli L, Stein DJ, Koenen KC, McLaughlin KA. Epidemiology of posttraumatic stress disorder: prevalence, correlates and consequences. Curr Opin Psychiatry. 2015;28(4):307-11.

39. Li X, Li S, Xiang M, Fang Y, Qian K, Xu J, et al. The prevalence and risk factors of PTSD symptoms among medical assistance workers during the COVID19 pandemic. J Psychosom Res. 2020;139:110270.

40. Wang Y, Ma S, Yang C, Cai Z, Hu S, Zhang B, et al. Acute psychological effects of Coronavirus Disease 2019 outbreak among healthcare workers in China: a cross-sectional study. Transl Psychiatry. 2020;10(1):348.

41. Stander VA, Thomsen CJ, Highfill-McRoy RM. Etiology of depression comorbidity in combat-related PTSD: a review of the literature. Clin Psychol Rev. 2014;34(2):87-98.

42. Wright KM, Britt TW, Bliese PD, Adler AB, Picchioni D, Moore D. Insomnia as predictor versus outcome of PTSD and depression among Iraq combat veterans. J Clin Psychol. 2011;67(12):1240-58.

43. Yang. Y, Li. W, Zhang. Q, Zhang. L, Cheung. T, Xiang. Y-T. Mental health services for older adults in China during the COVID-19 outbreak. Lancet Psychiatry. 2020;7(4):e19.

44. Kerai SM, Khan UR, Islam M, Asad N, Razzak J, Pasha O. Post-traumatic stress disorder and its predictors in emergency medical service personnel: a cross-sectional study from Karachi, Pakistan. BMC Emerg Med. 2017;17(1):26.

45. Lee SM, Kang WS, Cho AR, Kim T, Park JK. Psychological impact of the 2015 MERS outbreak on hospital workers and quarantined hemodialysis patients. Compr Psychiatry. 2018;87:123-7.

46. Matsumoto N, Kawaguchi J. Negative item memory and associative memory: Influences of working memory capacity, anxiety sensitivity, and looming cognition. J Behav Ther Exp Psychiatry. 2020;68:101569.

47. Lee D. The convergent, discriminant, and nomological validity of the Depression Anxiety Stress Scales-21 (DASS-21). J Affect Disord. $2019 ; 259: 136-42$.

48. Zou S, Liu ZH, Yan X, Wang H, Li Y, Xu X, et al. Prevalence and correlates of fatigue and its association with quality of life among clinically stable older psychiatric patients during the COVID-19 outbreak: a cross-sectional study. Global Health. 2020;16(1):119.

49. Liu CH, Zhang E, Wong GTF, Hyun S, Hahm HC. Factors associated with depression, anxiety, and PTSD symptomatology during the COVID-19 pandemic: Clinical implications for U.S. young adult mental health. Psychiatry Res. 2020;290:113172.

\section{Figures}




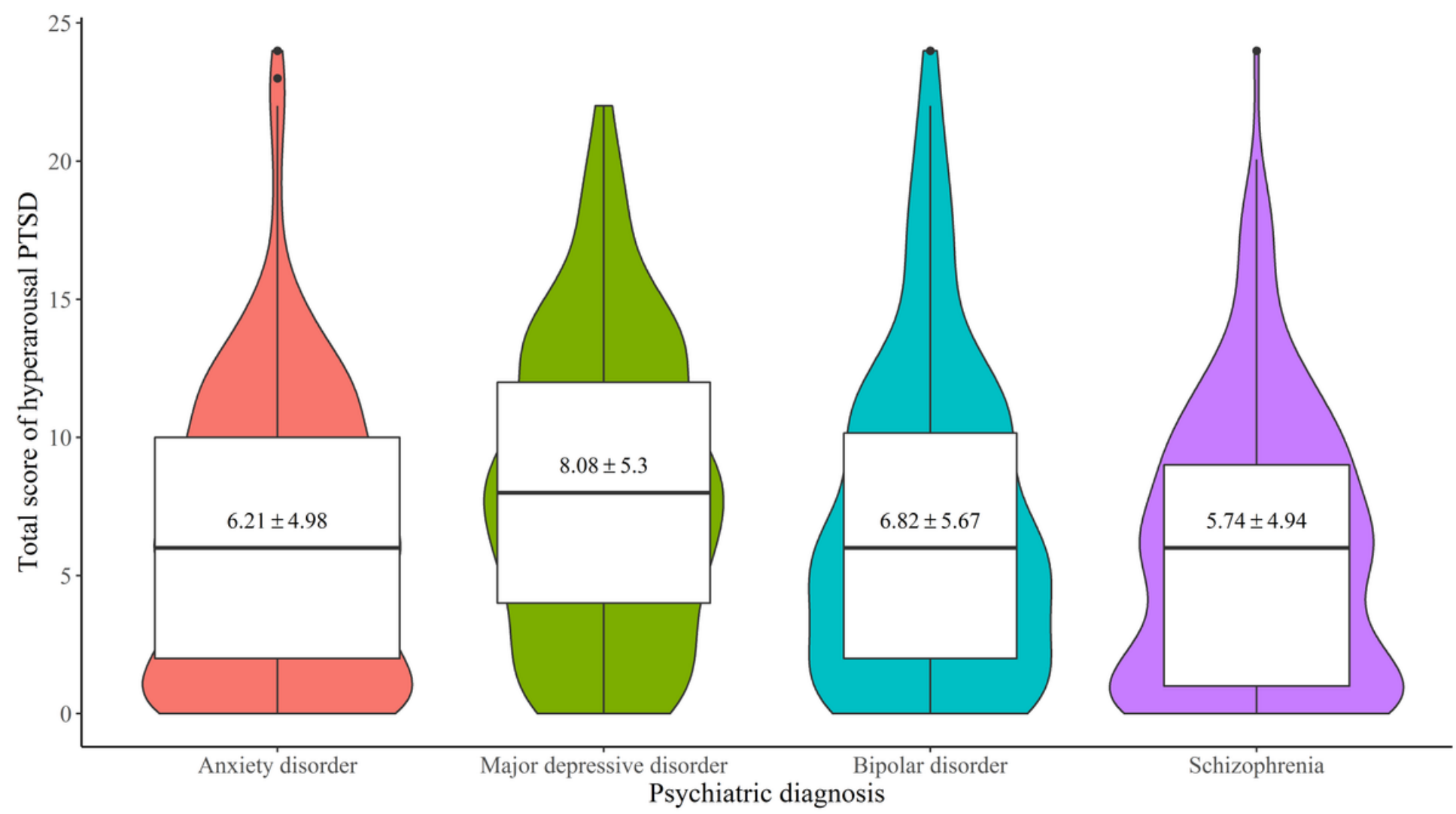

Figure 1

Total scores of hyperarousal symptom of PTSD among patients with different psychiatric diagnosis Note: The width of the figures indicates the sample size of each group. 